\title{
Aberrant Apolipoprotein E Expression and Cognitive Dysfunction in Patients with Poststroke Depression
}

\author{
Zhaohui Zhang, ${ }^{1,2}$ Junlin Mu, ${ }^{1,2}$ Jing Li, Wenqiang Li, ${ }^{1,2}$ and Jinggui Song ${ }^{1,2}$
}

Background: Apolipoprotein E (ApoE) is associated with some diseases with cognitive function defect. Aims: The purpose of this study was to examine the influence of ApoE on poststroke depression (PSD) risk and to define objective markers for diagnosis. Methods: The cognitive function, serum ApoE, and peripheral mononuclear blood cell ApoE mRNA expression of patients with PSD were compared to age-matched control patients with stroke and healthy volunteers. Sixty-seven patients with stroke were selected according to the cerebral infarction diagnosis standard of the Fourth National Cerebrovascular Disease Conference and divided into a PSD group (28 patients, 43-76 years old) or a control stroke group (39 patients, 43-78 years old) using the Hamilton Rating Scale for Depression, and compared to 40 healthy volunteers (42-78 years old). Cognitive function was evaluated by analysis of event-related potentials (ERPs), while expression of ApoE mRNA was determined by quantitative reverse transcription-polymerase chain reaction and serum ApoE by ELISA. Results: The latencies of ERP components N2 and P3 were prolonged, and the P3 amplitude was lower in the PSD group compared to the control stroke group and healthy controls $(p<0.01)$. There were no significant group differences in N1 and P2 latencies (all $p>0.05)$. The latency of N2 was positively correlated to the P3 latency in the PSD group $(p<0.05)$. No associations were detected between P3 amplitude, expression of ApoE mRNA, and serum ApoE in the PSD group (all $p>0.05$ ). The ERP results indicated that patients with PSD were significantly slower at identifying a target stimulus, suggesting deficits in perception and/or cognitive processing. Peripheral expression of ApoE mRNA was lower in the PSD group than the control stroke group $(p<0.701)$ while serum ApoE was higher than in the control stroke group $(p<0.05)$, possibly reflecting a feedback reduction in expression. Conclusion: We suggest that aberrant serum ApoE together with abnormalities in some ERP components may be useful markers for assessment of PSD risk and clinical diagnosis.

\section{Introduction}

A $S$ THE POPUlation AgEs, the incidence of stroke and stroke-related disability will likely rise, placing a significant strain on the public healthcare system. Depression after stroke (poststroke depression or PSD) (Tamam et al., 2009) is manifested by chronically depressed mood, lack of interest in daily activities, memory loss, sleep disorders, and cognitive impairment (Heide, et al., 2009) that reduced daily function and impede poststroke therapy. Abnormal lipid metabolism is closely correlated with stroke risk and depression. Apolipoprotein $\mathrm{E}(\mathrm{ApoE})$ is an intermediate-density lipoprotein that modulates other serum lipids by regulating the catabolism of triglycerides (Petkeviciene et al., 2012). The ApoE allele is associated with enhanced risk of cardiovascular diseases, including atherosclerosis and stroke (Winkler et al., 2010; Zhang et al., 2012), as well as Alzheimer's disease (AD). Furthermore, the ApoE polymorphisms are associated with depression in patients with AD (Bicalho et al., 2012), suggesting that aberrant ApoE may contribute to brain organic mental disorders. Although recent studies have identified the ApoE4 allele as the main genetic risk factor for AD (Kim et al., 2009) and linked ApoE polymorphisms to cognitive deficits (Hollingworth et al., 2011), little is known about the relationship between ApoE function and poststroke morbidity, including cognitive deficits and depression. We measured serum ApoE, peripheral ApoE expression, and cognitive processing capacity in patients with PSD, nondepressed stroke patients, and age-matched healthy controls to further investigate the correlation between ApoE and PSD and associated symptoms. The

\footnotetext{
${ }^{1}$ Department of Psychosomatic Medicine, The Second Affiliated Hospital of Xinxiang Medical University, Xinxiang, China.

${ }^{2}$ Henan Key Lab of Biological Psychiatry, Xinxiang Medical University, Xinxiang, China.

${ }^{3}$ Department of Neurology, The Third Affiliated Hospital of Xinxiang Medical University, Xinxiang, China.
} 
correlation between aberrant ApoE expression and cognitive dysfunction in patients with PSD as revealed by event-related potential (ERP) analysis defines these indices as possible markers for PSD risk or clinical diagnosis.

\section{Materials and Methods}

\section{Patients}

From January to October 2009, we selected 67 patients with stroke, all Xinxiang natives of Han ethnicity, from the Henan Provincial Mental Hospital according to the cerebral infarction diagnosis standard of the Fourth National Cerebrovascular Disease Conference. A poststroke depression group (28 patients, 43-76 years old) was distinguished from the nondepressed stroke patients (39 patients, 43-78 years old) using the Hamilton Rating Scale for Depression. A cohort of 40 healthy volunteers served as an age-matched control group (age range, 42-78 years old) and had no history of hypertension, cardiovascular diseases, or diabetes. There were no statistically significant differences in gender ratio, age, or other demographic variables such as the level of formal education between groups $(p>0.05)$.

\section{Measurement of event-related potential}

Both the PSD and nondepressed stroke groups were tested for cognitive function 2 weeks after stroke using the Dundee 4 Keypoint test. Event-related potentials (ERPs) were measured in a shielded room with patients lying relaxed, but alert. A reference electrode was placed in the right ear (A2), a ground on the forehead $(\mathrm{FPz})$, and recording electrodes $(\mathrm{Cz})$ placed along the central line according to the international brain electrical 10/20 system. Impedance between electrodes was $<5 \mathrm{~K} \Omega$, and ERPs were recorded for $600 \mathrm{~ms}$ after stimulus presentation. Test (target) stimuli consisted of $90-\mathrm{dB}, 4000-\mathrm{Hz}$ tones (20\% probability) interspersed between $80 \mathrm{~dB}, 1000 \mathrm{~Hz}$ nontarget stimuli ( $80 \%$ probability). Before experiments, the demand and instruction were told to the participant. The participant was requested to focus his/her attention on listening to the voice, and respond to target tones by the extension of the index finger of the superior hand. After the participant was confirmed to already fully grasp the main point, formal experiments procedure was performed. Participants were required to press a button in response to presentation of target stimuli, and reaction time was recorded in addition to ERPs. The data analyzed were the average response of two separate blocks of two trials.

\section{Measurement of ApoE mRNA expression in peripheral mononuclear blood cells by quantitative reverse transcription-polymerase chain reaction}

The primers for ApoE mRNA reverse transcription were designed using Primer 6.0 (F, 5'-GGGTCGCTTTTGGGAT TACCTG-3' and R, 5' ${ }^{\prime}$-CAACTCCTTCATGGTCTCGTCC-3'). The GAPDH gene was used as the internal references $\left(\mathrm{F}, 5^{\prime}\right.$ ACCACAGTCCATGCCATCAC-3'). Total RNA was extracted from mononuclear blood cells isolated using Takara's Trizol reagent and reverse transcribed into cDNA using Takara's kit according to the manufacturer's instructions. The reverse transcription-polymerase chain reaction was carried out in a $25-\mu \mathrm{L}$ reaction mixture containing of $12.5 \mu \mathrm{L}$ SYBRII master mix, $1 \mu \mathrm{L}$ of each primer, $6 \mu \mathrm{L}$ cDNA, and $4.5 \mu \mathrm{L}$ double-distilled water. The cycling conditions were an initial predenaturation step of $95^{\circ} \mathrm{C}$ for $3 \mathrm{~min}$, followed by 40 cycles of $25 \mathrm{~s}$ denaturation at $95^{\circ} \mathrm{C}$, annealing at $60^{\circ} \mathrm{C}$ for $30 \mathrm{~s}$, and extension at $72^{\circ} \mathrm{C}$ for $1 \mathrm{~min}$, and a final extension at $72^{\circ} \mathrm{C}$ for $10 \mathrm{~min}$. We analyzed the relative expression using the ratio, (Reference gene Ct value)/(Target gene Ct value).

\section{Measuring serum ApoE}

Serum ApoE was evaluated using an ELISA kit (R\&D Systems) according to the manufacturer's recommendations. Standard curve concentrations were calculated in triplicate for each plate.

\section{Statistic analysis}

All data are expressed as the mean \pm SE and were tested for normality and homogeneity of variance. Student's $t$-tests were used to compare paired group means, and one-way analysis of variance (ANOVA) was employed to compare differences among the three groups, followed by least significant difference post hoc pairwise comparisons. A $p<0.05$ was considered statistically significant.

\section{Results}

\section{Abnormal ERPs in patients with PSD}

As shown in Table 1, the poststimulus latencies of the N2 and P3 ERP components were significantly prolonged in patients with PSD compared to nondepressed stroke patients and healthy age-matched controls $\left(F_{\mathrm{N} 2}=152.516, p=0.008\right.$; $\left.F_{\mathrm{P} 3}=51.186, p=0.002\right)$, while the P3 amplitude was significantly reduced in patients with PSD compared to the other groups $\left(F_{\mathrm{P} 3}=40.633, p=0.005\right)$. In contrast, N1 and P2 latencies were not statistically different between groups $(p>0.05)$.

\section{Reduced expression of ApoE mRNA by patients with $P S D$}

Expression of ApoE mRNA was significantly lower in mononuclear blood cells in the PSD group $(0.77 \pm 0.24, n=27)$ than the control stroke group $(0.86 \pm 0.14, n=34)(t=2.854$, $p=0.006$ by $t$-test assuming unequal variance). One PSD specimen and five control stroke specimens were eliminated due to hemolysis.

\section{Elevated serum ApoE in patients with PSD}

The serum concentration of ApoE was significantly higher in the PSD group $[(0.99 \pm 0.23) \mathrm{mg} / \mathrm{dL}, n=27]$ than the control stroke group $[(0.85 \pm 0.28) \mathrm{mg} / \mathrm{dL}, n=34](t=2.014$, $p=0.048)$.

\section{Correlation analysis of N2 latency, P3 latency, P3 amplitude, ApoE mRNA expression, and serum ApoE between groups}

The scatter diagram of N2-versus-P3 latencies (N2PL and P3PL) is shown in Figure 1. The latency of N2 was positively correlated with P3 latency in the PSD group $(r=0.447$, $p=0.017$ ) (Table 2). In contrast, there were no significant associations between these waveform latencies and the other measured parameters or among P3 amplitude, ApoE mRNA expression, or serum ApoE concentration in patients with PSD (all $p>0.05)$. 
Table 1. Comparison of Event-Related Potentials Waveform-Component Latencies and P3 Amplitude Between Groups $(\bar{X} \pm s)$

\begin{tabular}{lccccc}
\hline Groups & N1PL $(m s)$ & P2PL $(m s)$ & N2PL $(m s)$ & P3PL $(m s)$ & P3amp $(\mu V)$ \\
\hline PSD group $(n=28)$ & $93.50 \pm 6.47$ & $175.75 \pm 13.82$ & $283.60 \pm 11.88^{\mathrm{a}}$ & $389.21 \pm 28.90^{\mathrm{a}}$ & $3.26 \pm 1.81^{\mathrm{b}}$ \\
Stroke group $(n=39)$ & $90.94 \pm 6.06$ & $179.91 \pm 11.89$ & $273.85 \pm 12.81$ & $368.00 \pm 36.70$ & $4.67 \pm 2.63$ \\
Control group $(n=40)$ & $90.84 \pm 6.81$ & $175.07 \pm 10.39$ & $236.03 \pm 11.42$ & $320.00 \pm 20.24$ & $8.63 \pm 2.94$ \\
$F$ & 1.646 & 1.670 & 152.516 & 51.186 & 40.633 \\
$P$ & 0.198 & 0.194 & 0.008 & 0.002 \\
\hline
\end{tabular}

${ }^{a} p<0.01$ compared to the nondepressed stroke group; $p<0.01$ compared to the healthy control group.

$\mathrm{b}_{p}<0.05$ compared to the nondepressed stroke group and $p<0.01$ compared with control group.

\section{Discussion}

Clinical depression is a major risk after stoke (Ferro et al., 2009). PSD may exacerbate cognitive dysfunction, impede neurological and functional recovery, and reduce patient quality of life (Brown et al., 2012), which in turn can increase the mortality or the risk of recurrence (Schmid et al., 2011). However, the signs of clinical depression can be difficult to distinguish from the short-term or long-term consequences of stroke. Thus, objective markers may greatly improve the accuracy of PSD diagnosis. ApoE is a critical regulator of lipid metabolism. Studies have shown that patients with PSD are more likely to exhibit dyslipidemia and aberrant serum ApoE concentration, a significant risk factor for cardiac and brain vascular diseases (Petkeviciene et al., 2012). Moreover, ApoE appears to influence the risk of cerebral infarction through effects on blood lipid metabolism (Saidi et al., 2007). At the same time, the ApoE genotype may independently contribute to the risk of depression. In the study, we sought to establish a correlation between peripheral ApoE gene expression, serum ApoE, and cognitive dysfunction to aid in clinical diagnosis and treatment of PSD.

ApoE binds to a specific receptor on liver cells and peripheral cells. It is essential for the normal catabolism of triglyceride-rich lipoprotein constituents and has a wellestablished role in blood cholesterol transport into the lymphatic system and then into the blood stream. Peripheral

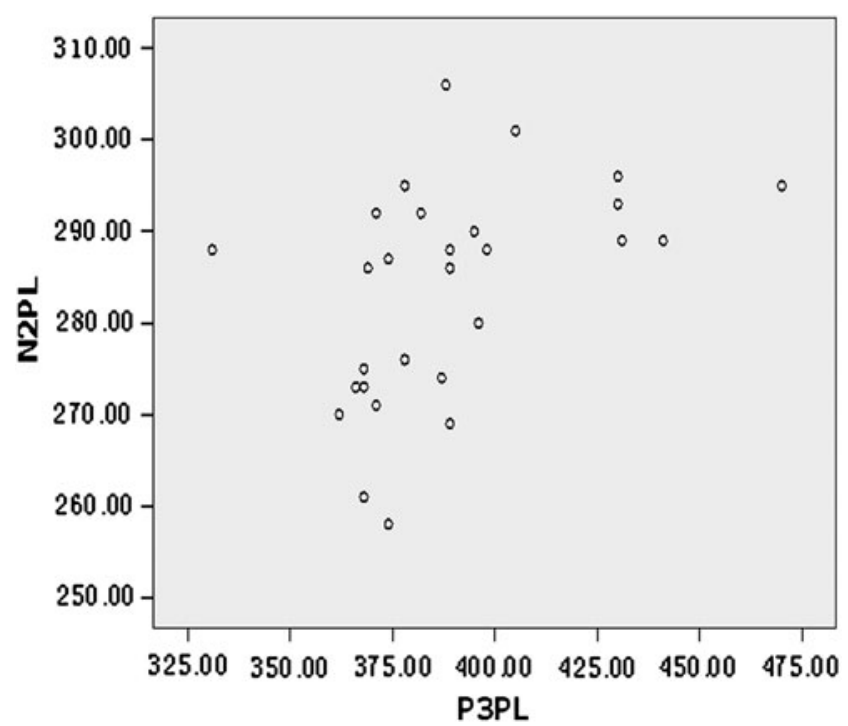

FIG. 1. Scatter diagram of N2PL and P3PL. (serum) ApoE is synthesized principally in the liver (Koob et al., 2010). In the nervous system, non-neuronal cell types, most notably astroglia and microglia, are the primary producers of ApoE, while neurons preferentially express the receptors for ApoE (Koob et al., 2010; Erdan et al., 2011). Recently, ApoE has been linked to several additional biological processes, including immunoregulation and cognition. Indeed, numerous studies have documented immunoregulation by ApoE, including the suppression of $\mathrm{T}$ cell proliferation, functional regulation of macrophages, facilitation of lipid antigen presentation to natural killer T cells, and modulation of oxidation and inflammation, processes central to diseases such as arteriosclerosis (McKay et al., 2011). Furthermore, expression of the ApoE4 allele is the best-known predictor for the progression of mild cognitive impairment (MCI) into AD (Sun et al., 2012) and so is predictive of age-related cognitive dysfunction (Kim et al., 2010). SpragueDawley rats performed poorly in maze tests after intrahippocampal injection of vectors containing the ApoE gene. Furthermore, this treatment was associated with neurodegeneration in the hippocampus (Eddins et al., 2009).

ERP is a noninvasive measure of early cognitive processing. ERPs include endogenous and exogenous compositions. Exogenous composition, including N1 and P2 waves with short latency, is affected by the physical characteristic of the stimuli. It is a reliable index for estimating the attention and cooperation of the subjects. Endogenous compositions, including N2 and P3 waves, which are greatly affected by psychological factors, are related to cognitive process. The latencies of N2 and P3 wave reflect the speed of the brain to encoding and classification and identification incident in the process of the brain identification; it also objectively reflects the advanced thinking activity such as cognitive function and judgment, but the amplitude of P3 indicates the testes' ability of assimilating information. The latency between stimulus

Table 2. Correlations Between all Parameters Measured $(R)$

\begin{tabular}{lccccc}
\hline Projects & N2PL & P3PL & P3amp & ApoE1 & ApoE2 \\
\hline N2PL & 1 & $0.447^{\mathrm{a}}$ & 0.145 & -0.061 & -0.255 \\
P3PL & $0.447^{\mathrm{a}}$ & 1 & 0.281 & -0.315 & -0.227 \\
P3amp & 0.145 & 0.281 & 1 & -0.052 & -0.053 \\
ApoE1 & -0.061 & -0.315 & -0.052 & 1 & 0.119 \\
ApoE2 & -0.255 & -0.227 & -0.053 & 0.119 & 1 \\
\hline
\end{tabular}

${ }^{a} p<0.05$.

ApoE1 indicates ApoE mRNA expression; ApoE2 indicates serum ApoE; $(R)$, correlation coefficient. 
presentation and the P300 (P3) waveform mainly reflects the time taken to evaluate or categorize a stimulus in a target/ nontarget series. In this study, we found that both the N2 and P3 latencies were prolonged in patients with PSD compared to nondepressed stroke patients and healthy controls $(p<0.01)$. In addition, the P3 amplitude was lower in patients with PSD $(p<0.01)$, and the latency of N2 was positively correlated to the latency of P3 $(p<0.05)$, indicating that patients with PSD were slower at identifying the target stimulus and suggesting a deficit in perception or judgment.

Patients with stroke are under stress, leading to overactivation of $\alpha_{1}$-NE receptors and resultant cognitive impairment. In normal circumstances, presynaptic $\alpha 2-\mathrm{NE}$ receptor agonists can restrain NE activity, and postsynaptic $\alpha 2-\mathrm{NE}$ receptor agonists can improve cognitive function. Our results support the opinion of Khedr (Khedr et al., 2009) that P300 can be used for evaluation of cognitive impairments in patients with PSD and possibly as a prognostic or diagnostic marker. Indeed, previous studies have demonstrated a relationship between P300 and cognitive impairment.

The P300 is associated with 5-HT and NE activity (Chen et al., 2005). An increase in P300 latency and a decrease in amplitude were associated with a reduction in 5-HT metabolic products in the cerebrospinal fluid. In the present study, the peripheral expression of ApoE mRNA was lower in the PSD group than in the nondepressed stroke group, while serum ApoE was significantly higher in the PSD group. A higher serum ApoE would reduce serum triglycerides, leading to a reduction in 5-HT concentration (Liang et al., 2009) and increased risk of PSD (Wang et al., 2009).

No association was detected among P3 amplitude, mononuclear cell ApoE mRNA expression, and serum ApoE concentration in the PSD group. It is possible that the influence of ApoE on lipid metabolism and cognitive function may be related to the specific allele. The ApoEc4 isoform can raise low-density lipoprotein $C$ and reduce serum TG, while ApoE\&2 has the opposite effect. Therefore, the relationship between ApoE and cognitive function may depend on the specific ApoE genotype. In addition, only the ApoE\&4 allele is a risk factor for cognitive decline, so the patients would exhibit varying levels of cognitive dysfunction after stroke.

In this study, our results demonstrate that a delayed P300, elevated serum ApoE, and reduced mononuclear cell ApoE expression are associated with depression after stroke.

\section{Acknowledgments}

The authors thank the patients, their families, and the healthy volunteers for their participation, and the physicians who helped us to take clinical data and blood samples in the Second Affiliated Hospital of Xinxiang Medical University. We thank Prof. Wei Gu (the Second Xiangya Hosptial of Central South University) and Lele Ma (State Key Laboratory of Biotherapy of Sichuan University), who assisted in revision of the manuscript. Funding for this study was provided by the Henan medical science research funded project (NO. 20080308), the Natural Science Foundation of Henan (No. $112300410165,122300413212)$, and Xinxiang medical university Highly Educated Projects (NO. 08BSSKYQD-003).

\section{Author Disclosure Statement}

No competing financial interests exist.

\section{References}

Bicalho MA, Pimenta FA, Bastos-Rodrigues L, et al. (2012) Sociodemographic characteristics, clinical factors, and genetic polymorphisms associated with Alzheimer's disease. Int J Geriatr Psychiatry. [Epub ahead of print]; DOI: 10.1002/ gps.3875.

Brown C, Hasson H, Thyselius V, et al. (2012) Post-stroke depression and functional independence: a conundrum. Acta Neurol Scand 126:45-51.

Chen TJ, Yu YW, Chen MC, et al. (2005) Serotonin dysfunction and suicide attempts in major depressives: an auditory eventrelated potential study. Neuropsychobiology 52:28-36.

Eddins D, Klein RC, Yakel JL, et al. (2009) Hippocampal infusions of apolipoprotein E peptides induce long-lasting cognitive impairment. Brain Res Bull 79:111-115.

Erdan Sun, Apiradee Lim, Xipu Liu, et al. (2011) Apolipoprotein $\mathrm{E}$ gene and age-related macular degeneration in a Chinese population. Molecular Vision 17:997-1102.

Ferro JM, Caeiro L, Santos C. (2009) Poststroke emotional and behavior impairment:a narrative review. Cerebrovasc Dis 27:197-203.

Heide S, Manfred K, Gläser C, et al. (2009) Apolipoprotein E (apoE) polymorphism: a risk factor for fatal coronary sclerosis. Forensic Sci Int 192:62-66.

Hollingworth P, Sweet R, Sims R, et al. (2011) Genome-wide association study of Alzheimer's disease with psychotic symptoms. Mol Psychiatry. [Epub ahead of print]; DOI: 10.1038/ mp.2011.125.

Khedr EM, Hamed SA, El-Shereef HK, et al. (2009) Cognitive impairment after cerebrovascular stroke: Relationship to vascular risk factors [J]. Neuropsychiatr Dis Treat 5:103116.

Kim J, Basak JM, Holtzman DM. (2009) The Role of Apolipoprotein E in Alzheimer's disease. Neuron 63:287-303.

Kim JM, Kim SY, Bae KY, et al. (2010) Apolipoprotein E4 genotype and depressive symptoms as risk factors for dementia in an older korean population. Psychiatry Invest 7:135-140.

Koob AO, Paulini AD, Masliah E. (2010) GFAP reactivity, apolipoprotein E redistribution and cholesterol reduction in human astrocytes treated with alpha-synuclein. Neurosci Lett 469:11-14.

Liang S, Pan M, Geng HH, et al. (2009) Apolipoprotein E polymorphism in normal Han Chinese population: frequency and effect on lipid parameters. Mol Biol Rep 36:1251-1256.

McKay GJ, Silvestri G, Chakravarthy U, et al. (2011) Variations in apolipoprotein E frequency with age in a pooled analysis of a large group of older people. Am J Epidemiol 173:13571364.

Petkeviciene J, Smalinskiene A, Luksiene DI, et al. (2012) Associations between Apolipoprotein E genotype, diet, body mass index, and serum lipids in lithuanian adult population. PLoS One 7:e41525.

Saidi S, Slamia LB, Ammou SB, et al. (2007) Association of apolipoprotein $\mathrm{E}$ gene polymorphism with ischemic stroke involving large-vessel disease and its relation to serum lipid levels. J Stroke Cerebrovasc Dis 16:160-166.

Schmid AA, Kroenke K, Hendrie HC, et al. (2011) Poststroke depression and treatment effects on functional outcomes. Neurology 76:1000-1005.

Sun X, Nicholas J, Walker A, et al. (2012) APOE genotype in the diagnosis of Alzheimer's disease in patients with cognitive impairment. Am J Alzheimers Dis Other Demen 27: 315-320. 
Tamam Y, Tasdemir N, Toprak R, et al. (2009) Apolipoprotein $\mathrm{E}$ genotype in patients with cerebrovascular diseases and its effect on the disease outcome. Int J Neurosci 119: 919-935.

Wang SH, Zhang ZJ, Guo YJ, et al. (2009) Expression of Decreased expression of serotonin $1 \mathrm{~A}$ receptor in the dentate gyrus in association with chronic mild stress: a rat model of post-stroke depression. Psychiatry Res 170:245-251.

Winkler K, Hoffmann MM, Krane V, et al. (2010) Apolipoprotein E genotype predicts cardiovascular endpoints in dialysis patients with type 2 diabetes mellitus. Atherosclerosis 208:197202.

Zhang RJ, Wang XF, Liu JX, et al. (2012) Apolipoprotein E Gene Polymorphism and the Risk of Intracerebral Hemorrhage in the Chinese Population. Genet Test Mol Bioma 16:63-66.
Address correspondence to: Jinggui Song, M.D.

Department of Psychosomatic Medicine The Second Affiliated Hospital of Xinxiang Medical University

Xinxiang, Henan 453002

China

E-mail: songjg62@126.com

Wenqiang Li, M.Sc. Henan Key Lab of Biological Psychiatry Xinxiang Medical University No. 388, Jianshe Middle Road Xinxiang 453002

China

E-mail: lwq781603@163.com 